\title{
RIGIDITY OF HYPERBOLIC P-MANIFOLDS: A SURVEY.
}

\author{
JEAN-FRANÇOIS LAFONT
}

\begin{abstract}
In this survey paper, we outline the proofs of the rigidity results (Mostow type, quasi-isometric, and Diagram rigidity) for simple, thick, hyperbolic P-manifolds found in [L1], [L2], [L3]. The later spaces are in some sense the simplest non-manifold locally CAT(1) spaces one can create. All the proofs depend on the highly non-homogenous structure of the boundary at infinity of the (universal covers of the) spaces in question. We emphasize the similarities and differences in the proofs of the various rigidity results.
\end{abstract}

\section{INTRODUCTION.}

In recent years, there has been much interest in proving rigidity type theorems for nonpositively curved spaces. All of these results originated from Mostow's seminal work $[\mathrm{M}]$, in which he showed that homotopy equivalent compact, rank one, locally-symmetric spaces of non-compact type are always isometric.

In the series of papers [L1], [L2], [L3], the author exhibited rigidity results for a particularly nice class of singular spaces. The spaces in question, called hyperbolic P-manifolds, are in some sense the simplest non-manifold CAT(-1) spaces one can consider. Let us start by defining these spaces:

Definition 1.1. A closed $n$-dimensional piecewise manifold (henceforth abbreviated to Pmanifold) is a topological space which has a natural stratification into pieces which are manifolds. More precisely, we define a 1-dimensional P-manifold to be a finite graph. An $n$ dimensional P-manifold $(n \geq 2)$ is defined inductively as a closed pair $X_{n-1} \subset X_{n}$ satisfying the following conditions:

- Each connected component of $X_{n-1}$ is either an $(n-1)$-dimensional P-manifold, or an $(n-1)$-dimensional manifold.

- The closure of each connected component of $X_{n}-X_{n-1}$ is homeomorphic to a compact orientable $n$-manifold with boundary, and the homeomorphism takes the component of $X_{n}-X_{n-1}$ to the interior of the $n$-manifold with boundary; the closure of such a component will be called a chamber.

Denoting the closures of the connected components of $X_{n}-X_{n-1}$ by $W_{i}$, we observe that we have a natural map $\rho: \coprod \partial W_{i} \longrightarrow X_{n-1}$ from the disjoint union of the boundary components of the chambers to the subspace $X_{n-1}$. We also require this map to be surjective, and a

Key words and phrases. Mostow Rigidity, quasi-isometry, diagram of groups, singular spaces, CAT(-1), hyperbolic groups. 
homeomorphism when restricted to each component. The P-manifold is said to be thick provided that each point in $X_{n-1}$ has at least three pre-images under $\rho$. We will henceforth use a superscript $X^{n}$ to refer to an $n$-dimensional P-manifold, and will reserve the use of subscripts $X_{n-1}, \ldots, X_{1}$ to refer to the lower dimensional strata. For a thick $n$-dimensional P-manifold, we will call the $X_{n-1}$ strata the branching locus of the P-manifold.

Intuitively, we can think of P-manifolds as being "built" by gluing manifolds with boundary together along lower dimensional pieces. Examples of P-manifolds include finite graphs and soap bubble clusters. Observe that compact manifolds can also be viewed as (non-thick) P-manifolds. Less trivial examples can be constructed more or less arbitrarily by finding families of manifolds with homeomorphic boundary and glueing them together along the boundary using arbitrary homeomorphisms. We now define the family of metrics we are interested in.

Definition 1.2. A Riemannian metric on a 1-dimensional P-manifold (finite graph) is merely a length function on the edge set. A Riemannian metric on an $n$-dimensional P-manifold $X^{n}$ is obtained by first building a Riemannian metric on the $X_{n-1}$ subspace, then picking, for each $W_{i}$ a Riemannian metric with totally geodesic boundary satisfying that the gluing map $\rho$ is an isometry. We say that a Riemannian metric on a P-manifold is hyperbolic if at each step, the metric on each $W_{i}$ is hyperbolic.

A hyperbolic P-manifold $X^{n}$ is automatically a locally $C A T(-1)$ space (see Chapter II.11 in Bridson-Haefliger $[\mathrm{BH}]$ ). Furthermore, the lower dimensional strata $X_{i}$ are totally geodesic subspaces of $X^{n}$. In particular, the universal cover $\tilde{X}^{n}$ of a hyperbolic P-manifold $X^{n}$ is a $C A T(-1)$ space (so is automatically $\delta$-hyperbolic), and has a well-defined boundary at infinity $\partial^{\infty} \tilde{X}^{n}$. Finally we note that the fundamental group $\pi_{1}\left(X_{n}\right)$ is a $\delta$-hyperbolic group. We refer the reader to $[\mathrm{BH}]$ for background on $C A T(-1)$ and $\delta$-hyperbolic spaces.

Definition 1.3. We say that an $n$-dimensional P-manifold $X^{n}$ is simple provided its codimension two strata is empty. In other words, the $(n-1)$-dimensional strata $X_{n-1}$ consists of a disjoint union of $(n-1)$-dimensional manifolds. We further assume that for each chamber, the various boundary components get attached to distinct components of the codimension one strata.

Examples of simple, thick, hyperbolic P-manifolds can easily be constructed via arithmetic techniques. The rigidity results contained in the papers [L1], [L2], [L3] can now be summarized in the following:

Theorem 1.4. Let $X^{n}$ be an $n$-dimensional, simple, thick, hyperbolic P-manifold. Then we have:

- if $n=2$, then $X^{n}$ is topologically rigid.

- if $n \geq 3$, then $X^{n}$ is Mostow rigid.

- if $n \geq 3$, then $\pi_{1}\left(X^{n}\right)$ is quasi-isometrically rigid. 
In particular, if $n \geq 2$, the class of fundamental groups of $n$-dimensional, simple, thick, hyperbolic P-manifolds exhibits diagram rigidity.

Let us briefly recall the terms involved. By topological rigidity, we mean that if $X_{1}^{2}$ and $X_{2}^{2}$ have isomorphic fundamental group, then they are in fact homeomorphic. By Mostow rigidity, we mean that if $X_{1}^{n}$ and $X_{2}^{n}$ have isomorphic fundamental group, then they are in fact isometric. Quasi-isometric rigidity, refers to the fact that the only groups which are quasi-isometric to $\pi_{1}\left(X^{n}\right)$ are, up to finite extension, uniform lattices in $I \operatorname{som}\left(\tilde{X}^{n}\right)$. Finally, observe that the generalized Seifert-Van Kampen theorem allows us to write the fundamental group of any $n$-dimensional, simple, thick, hyperbolic P-manifold as the direct limit of a canonical diagram of groups. Diagram rigidity now refers to the fact that two such direct limits are isomorphic if and only if their diagrams are isomorphic.

The proof of these results naturally breaks down into two steps. The first step requires an analysis of the topology of the boundary at infinity of the universal cover of the simple, thick, hyperbolic P-manifolds. The second step 'pushes back' the information garnered at the boundary at infinity into the universal cover.

We will use the following standard notation: $S^{n}$ refers to an $n$-dimensional sphere, $\mathbb{D}^{n}$ to a closed $n$-dimensional ball, and $\mathbb{D}_{\circ}^{n}$ to an open $n$-dimensional ball. $\mathbb{H}^{n}$ refers to the standard hyperbolic space of constant curvature -1 .

\section{Topology of the Boundary AT INFinity.}

We start by noting that, in a simple, thick, hyperbolic P-manifold $X^{n}$, there is a codimension one singular set $B$ which consists of a union of closed hyperbolic $(n-1)$-dimensional manifolds. Let $\mathcal{B}$ denote the full pre-image of the singular set in the universal cover $\tilde{X}^{n}$. Observe that each connected component $\tilde{B}_{j}$ of $\mathcal{B}$, lies as a totally geodesic subspace (isometric to $\mathbb{H}^{n-1}$ ) of the universal cover. This implies that the boundary at infinity of $\tilde{B}_{j}$ (homeomorphic to $S^{n-2}$ ) naturally embeds in the boundary at infinity of $\tilde{X}^{n}$. By abuse of notation, let $\partial^{\infty} \mathcal{B}$ denote the subset of $\partial^{\infty} \tilde{X}^{n}$ consisting of the union of all the boundaries at infinity of the various connected components of the branching locus, i.e. $\partial^{\infty} \mathcal{B}=\bigcup_{j} \partial^{\infty} \tilde{B}_{j}$.

Theorem 2.1. Let $\partial^{\infty} \tilde{X}_{1}^{n}, \partial^{\infty} \tilde{X}_{2}^{n}(n \geq 2)$ be the boundary at infinity of the universal cover of a pair of simple, thick, hyperbolic P-manifold $X_{i}^{n}$, and let $\phi: \partial^{\infty} \tilde{X}_{1}^{n} \rightarrow \partial^{\infty} \tilde{X}_{2}^{n}$ be an arbitrary homeomorphism. Then for each connected component $\tilde{B}_{1, j}$ in $\mathcal{B}_{1}$, there is a connected component $\tilde{B}_{2, j^{\prime}}$ in $\mathcal{B}_{2}$ with the property that $\phi\left(\partial^{\infty} \tilde{B}_{1, j}\right)=\partial^{\infty} \tilde{B}_{2, j^{\prime}}$.

In order to show this result, the natural starting point is to obtain a topological characterization of points lying in $\partial^{\infty} \mathcal{B}$ that will distinguish them from points that are not in $\partial^{\infty} \mathcal{B}$. This motivated the notion of $n$-branching, defined as follows:

Definition 2.2. Define the 1-tripod $T$ to be the topological space obtained by taking the join of a one point set with a three point set. Denote by $*$ the point in $T$ corresponding to the one point set. We define the $n$-tripod $(n \geq 2)$ to be the space $T \times \mathbb{D}^{n-1}$, and call the 
subset $* \times \mathbb{D}^{n-1}$ the spine of the tripod $T \times \mathbb{D}^{n-1}$. The subset $* \times \mathbb{D}^{n-1}$ separates $T \times \mathbb{D}^{n-1}$ into three open sets, which we call the open leaves of the tripod. The union of an open leaf with the spine will be called a closed leaf of the tripod. We say that a point $p$ in a topological space $X$ is $n$-branching provided there is a topological embedding $f: T \times \mathbb{D}^{n-1} \longrightarrow X$ such that $p \in f\left(* \times \mathbb{D}_{\circ}^{n-1}\right)$.

The topological characterization of points lying in $\mathcal{B}$ is contained in the following:

Proposition 2.3. Let $X^{n}$ be a simple, thick, hyperbolic P-manifold $(n \geq 2), \partial^{\infty} \tilde{X}^{n}$ the boundary at infinity of its universal cover. Then $p \in \partial^{\infty} \tilde{X}^{n}$ is $(n-1)$-branching if and only if $p \in \partial^{\infty} \mathcal{B}$.

We will first explain how Theorem 2.1 follows from Proposition 2.2, and then proceed to explain the argument behind Proposition 2.2.

2.1. Reducing the Theorem to the Proposition. Let us briefly explain how Theorem 2.1 can be obtained from Proposition 2.2. We first observe that the property of being $(n-1)$-branching is clearly a topological invariant. So the proposition implies that, if $\phi: \partial^{\infty} \tilde{X}_{1}^{n} \rightarrow \partial^{\infty} \tilde{X}_{2}^{n}$ is an arbitrary homomorphism, we have that $\phi\left(\partial^{\infty} \mathcal{B}_{1}\right)=\partial^{\infty} \mathcal{B}_{2}$.

Now recall that $\mathcal{B}_{i}=\bigcup_{j} \partial^{\infty} \tilde{B}_{i, j}$. It is easy to see that each $\partial^{\infty} \tilde{B}_{i, j}$ is a closed subset of $\partial^{\infty} \tilde{X}_{i}^{n}$, that they are pairwise disjoint, and furthermore if $n \geq 3$ that each $\partial^{\infty} \tilde{B}_{i, j}$ is connected (being homeomorphic to $S^{n-2}$ ). So if $n \geq 3$, we can now apply a result of Sierpinski [S] that states the following: let $X$ be an arbitrary topological space, $\left\{C_{i}\right\}$ a countable collection of disjoint path connected closed subsets in $X$. Then the path connected components of $\cup C_{i}$ are precisely the individual $C_{i}$. Applying this to the subset $\partial^{\infty} \mathcal{B}_{i}$ in $\partial^{\infty} \tilde{X}_{i}^{n}$, we see that the path-connected components of $\partial^{\infty} \mathcal{B}_{i}$ are precisely the individual $\partial^{\infty} \tilde{B}_{i, j}$. This forces the map $\phi$ to take each $\partial^{\infty} \tilde{B}_{1, j}$ homeomorphically onto some $\partial^{\infty} \tilde{B}_{2, j^{\prime}}$, which is the statement in Theorem 2.1.

In the case where $n=2$, one needs to be a bit more careful. The problem here is that when $n=2$, the branching locus in $X_{i}^{2}$ is 1-dimensional, i.e. consists of a finite union of closed geodesics. This implies that each $\partial^{\infty} \tilde{B}_{i, j}$ in fact consists of just a pair of points (i.e. an $S^{0}$ ), corresponding to the two endpoints of a geodesic ray (a lift of the closed geodesic). In particular, in this case, each subset $\partial^{\infty} \tilde{B}_{i, j}$ is not connected, and so Sierpinski's result is of no use. In order to get around this, we refine the information we have in the 2-dimensional setting by considering separation properties of pairs of points in $\partial^{\infty} \tilde{X}_{i}^{2}$. Namely, one shows the following:

Proposition 2.4. Let $\partial^{\infty} \tilde{X}^{2}$ be the boundary at infinity of the universal cover of a simple, thick, hyperbolic P-manifold of dimension 2. Then for a pair of points $x, y \in \partial^{\infty} \tilde{X}^{2}$, the following statements are equivalent:

- $\partial^{\infty} \tilde{X}^{2}-\{x, y\}$ has at least three path-connected components.

- $\partial^{\infty} \tilde{B}_{j}=\{x, y\}$ for some connected component $B_{j}$ in $\mathcal{B}$. 
The argument for this Proposition relies on the fact that $\partial^{\infty} \tilde{X}^{2}$ contains many isometrically embedded $\mathbb{H}^{2}$ (this is ensured by the thickness hypothesis). Each of these yields an embedded $S^{1}$ in the boundary at infinity, allowing us to construct paths between points in the boundary at infinity by concatenating paths traveling along the various $S^{1}$ 's. The details of the proof can be found in [L3], and constitute the main difference between the 2-dimensional case and the higher dimensional cases.

2.2. The argument for the Proposition. We now proceed to explain how to prove Proposition 2.2. Recall that the Proposition states that points in $\partial^{\infty} \tilde{X}^{n}$ are $(n-1)$-branching precisely if they lie in some $\partial^{\infty} \tilde{B}_{j}$.

Well one direction of the implication is relatively easy: to show that each point in $\partial^{\infty} \tilde{B}_{j}$ is $(n-1)$-branching, we start by observing that $\tilde{B}_{j}$, being a connected component of the lift of the branching locus, is in fact an isometrically embedded $\mathbb{H}^{n-1}$ (this follows immediately from the simplicity assumption), and hence that $\partial^{\infty} \tilde{B}_{j}$ is homeomorphic to an $S^{n-2}$.

Furthermore, it is easy to show that there exist, inside $\tilde{X}^{n}$, three isometrically embedded "half $\mathbb{H}^{n}$ 's", with the property that their common boundary is $\tilde{B}_{j}$ (this follows from the thickness hypothesis). The net effect is that, on the level of the boundary at infinity, one can find three disjoint embedded $(n-1)$-dimensional closed disks $\mathbb{D}^{n-1}$ with the property that their boundaries all map homeomorphically to the $\partial^{\infty} \tilde{B}_{j}$ (an embedded $S^{n-2}$ ). But this immediately gives you that the points in $\partial^{\infty} \tilde{B}_{j}$ are $(n-1)$-branching, completing one of the desired implications.

The reverse implication is considerably harder. One wants to argue that if $p \in \partial^{\infty} \tilde{X}^{n}$ does not lie in one of the $\partial^{\infty} \tilde{B}_{j}$, then $p$ is not $(n-1)$-branching. So pick a point that does not lie in $\partial^{\infty} \tilde{B}_{j}$. With some work, one can argue that it is suffices to assume that $p$ lies in $\partial^{\infty} \tilde{W}$ where $\tilde{W}$ is a connected lift of one of the chambers. We now would like to prove that the point $p$ is not $(n-1)$-branching, i.e. to show that there are no injective maps $f: T \times \mathbb{D}^{n-2} \rightarrow \partial^{\infty} \tilde{X}^{n}$ satisfying $p \in f\left(* \times \mathbb{D}^{n-2}\right)$.

The immediate difficulty is that one does not know much about the topology of $\partial^{\infty} \tilde{X}^{n}$. But one of the nice properties of CAT $(-1)$ spaces is the existence of continuous maps from the boundary at infinity to the link of any point inside the space. Recall that the link of a point essentially encodes the space of "directions" at that point. The key feature we will need is that the link of a point in the interior of $\tilde{W}$ is homeomorphic to $S^{n-1}$ (since such a point has a manifold neighborhood). If $\rho$ denotes the projection map, we can now study the composite map:

$$
\rho \circ f: T \times \mathbb{D}^{n-2} \longrightarrow \partial^{\infty} \tilde{X}^{n} \longrightarrow S^{n-1}
$$

Note that it is immediate that points in $S^{n-1}$ cannot be $(n-1)$-branching (for instance, they have the wrong local homology), and hence the composite map $\rho \circ f$ must fail to be injective.

The trick now boils down to ensuring that the composite map fails to be injective at a point $q$ in the target where the projection map $\rho$ is injective. This will immediately imply that 
the original map $f$ was not injective at the point $\rho^{-1}(q)$. So the next step is to understand the subset of $S^{n-1}$ where the map $\rho$ is injective. This is not too hard: an easy exercise in elementary hyperbolic geometry gives you that the set of injective points in $S^{n-1}$ consists of the complement of a countable dense set of disjoint open metric balls in $S^{n-1}$. Let us denote this set by $I$, and the subset consisting of the boundaries of the various open metric balls by $\partial I \subset I$ (note that this subset is not the boundary of the set $I$ in the topological sense).

One also has that the point $\rho(p)$ in fact lies in $I-\partial I$. Points inside $I-\partial I \subset S^{n-1}$ can be seen to have a very special property: if an open set $U \subset S^{n-1}$ contains one of these points in its closure, then $U$ must in fact contain a point in $I$. Since the set $I$ consists of those points where $\rho$ is injective, we have reduced the proof of Proposition 2.2 to the following:

Claim: Let $\rho \circ f: T \times \mathbb{D}^{n-2} \rightarrow S^{n-1}$ be as above. Then there exists an open set $U \subset S^{n-1}$ with the property that:

- for every point $y$ in $U,(\rho \circ f)^{-1}(y)$ consists of at least two points.

- the closure of $U$ contains $\rho(p)$.

Before discussing the general case, let us explain the Claim in the specific case where $n=2$. In this situation the argument is particularly easy: we have a map $\rho \circ f: T \rightarrow S^{1}$, and the set of points $I$ where the geodesic retraction $\rho$ is injective is actually a Cantor set embedded in $S^{1}$. Now observe that the set $I$ can be decomposed into two disjoint pieces: $\partial I$, consisting of the endpoints of the open intervals appearing in the creation of the Cantor set, and $I-\partial I$ consisting of the remaining points. The hypothesis forces $\rho(p) \in I-\partial I$, and in particular the composite map $\rho \circ f$ is injective at that point. Since we are inside $S^{1}$, the point $\rho(p)$ locally separates $S^{1}$ into two components, and we must have two of the three leaves of the tripod $T$ mapping to the same side of the point $\rho(p)$. Taking $U$ to be a small enough open interval in $S^{1}$ in the appropriate side, and containing $\rho(p)$ in its closure, this immediately implies the claim.

We now proceed to the general case, i.e. where $n \geq 3$. The first step is to establish that an entire neighborhood $V$ of $f^{-1}(p)$ inside the spine $* \times \mathbb{D}^{n-2}$ maps to the set $I$. Now consider the restriction of the composite map $\rho \circ f$ to each of the three closed leaves of $T \times \mathbb{D}^{n-2}$. Note that each of the closed leaves is homeomorphic to $\mathbb{D}^{n-1}$, with boundary homeomorphic to $S^{n-2}$. Hence the restriction of the composite to the boundary of each closed leaf yields a continuous map from $S^{n-2}$ into $S^{n-1}$. Furthermore, the three maps $F_{1}, F_{2}, F_{3}$ obtained from the three closed leaves have the property that they coincide on the spine, and in particular on the open subset $V$ of the spine. To conclude the argument for the claim, we would like to make precise the following heuristic argument:

(1) since the composite $\rho \circ f$ is injective on $V$, its image is a topological embedding of $\mathbb{D}^{n-2}$ inside $S^{n-1}$, and hence should "locally separate" $S^{n-1}$ into two components. 
(2) the restriction of $\rho \circ f$ to each of the three closed leaves must surject onto one of the two components, and hence one of the two components must lie in the image of two distinct closed leaves.

Note that this is an immediate analogue of what occurs in the simpler $n=2$ case.

In trying to make the above argument precise, one ends up involved in the study of the maps $F_{1}, F_{2}, F_{3}$, which are maps from $S^{n-2}$ into $S^{n-1}$ with the property that they are injective on the small open set $V$. The key property concerning these maps is the following strong form of the Jordan separation theorem:

Theorem 2.5. Let $f: S^{n-2} \rightarrow S^{n-1}$ be a continuous map, and assume that $f$ is injective on an open set $V \subset S^{n-2}$. Then:

- $f\left(S^{n-2}\right)$ separates $S^{n-1}$.

- there are precisely two connected components $U_{1}, U_{2}$ in $S^{n-1}-f\left(S^{n-2}\right)$ having the property that there closure intersects $f(V)$.

- if $F: \mathbb{D}^{n-1} \rightarrow S^{n-1}$ is any extension of the map $f$, then $F$ must surject onto either $U_{1}$ or $U_{2}$.

In the first paper [L1] in the series, Theorem 2.4 was shown in the case where $n=3$. Note that in this case, one is looking at maps from $S^{1}$ to $S^{2}$; the situation is then greatly simplified by the Schoenflies Theorem, which was the key to establishing Theorem 2.4 in the $n=3$ case.

When $n>3$, we know that the Schoenflies Theorem fails, and the argument used in [L1] has no chance of extending to higher dimensions. So in [L2], a different argument was used to establish Theorem 2.4. The argument relies on Alexander duality, local Betti numbers, as well as the sophisticated codimension one taming results of Bing [B], Ancel-Cannon [AC], and Ancel [A].

\section{Concluding the proofs.}

As we mentioned earlier, Theorem 2.1 is the key result towards proving the Main Theorem. We now proceed to explain how to conclude the proof of the Main Theorem.

3.1. The common feature. First of all, recall that quasi-isometries between $\delta$-hyperbolic spaces induce homeomorphisms between their boundaries at infinity. Note that an isomorphism between fundamental groups yield quasi-isometries of the universal cover.

Now Theorem 2.1 essentially tells you that if $X_{i}^{n}$ are a pair of simple, thick, hyperbolic P-manifolds, and if $\Gamma_{i}=\pi_{1}\left(X_{i}^{n}\right)$ are the two fundamental groups, then any isomorphism $\phi: \Gamma_{1} \rightarrow \Gamma_{2}$ naturally induces a bijection from the connected components of $\mathcal{B}_{1}$ to the connected components of $\mathcal{B}_{2}$.

The next step is to also obtain a bijection between the lifts of chambers in the respective $\tilde{X}_{i}^{n}$. In order to do this, we exploit the separation properties of the $\partial^{\infty} B_{i, j} \subset \partial^{\infty} \tilde{X}_{i}^{n}$. Depending on the dimension, we prove that: 
- if $n \geq 3$, each path-connected component of $\partial^{\infty} \tilde{X}^{n}-\partial^{\infty} \mathcal{B}$ containing at least two points corresponds canonically with a unique connected lift of a chamber in $\tilde{X}^{n}$.

- if $n=2$, we define an equivalence relation $\equiv$ on $\partial^{\infty} \tilde{X}^{2}-\partial^{\infty} \mathcal{B}$ by defining $x \equiv y$ provided $\partial^{\infty} \tilde{X}^{2}-\{x, y\}$ has precisely two connected components, and show that there is a canonical correspondance between equivalence classes $\mathcal{C}$ in $\partial^{\infty} \tilde{X}^{2}$ satisfying $|\mathcal{C}|>1$ on the one hand, and connected lifts of chambers in $\tilde{X}^{2}$ on the other.

Furthermore, in both cases above, if $W$ is the connected lift of a chamber associated to the subset $Y \subset \partial^{\infty} \tilde{X}^{n}-\partial^{\infty} \mathcal{B}$ (where $Y$ is as above, according to whether $n=2$ or $n \geq 3$ ), then we also have that $\partial^{\infty} W \subset \partial^{\infty} \tilde{X}^{n}$ coincides with the closure of $Y$ in $\partial^{\infty} \tilde{X}^{n}$.

The upshot is that the homeomorphism between the boundaries at infinity take boundaries of lifts of chambers in $\partial^{\infty} \tilde{X}_{1}^{n}$ to boundaries of lifts of chambers in $\partial^{\infty} \tilde{X}_{2}^{n}$.

3.2. Mostow and topological rigidity. At this point, we have that the isomorphism $\phi: \Gamma_{1} \rightarrow \Gamma_{2}$ induces bijections between:

- connected components of $\mathcal{B}_{1}$ and connected components of $\mathcal{B}_{2}$, and

- connected lifts of chambers in $\tilde{X}_{1}^{n}$ and connected lifts of chambers in $\tilde{X}_{2}^{n}$.

Furthermore, the bijections are compatible, in the sense that if a connected component of $\mathcal{B}_{1}$ is contained in the connected lift of a chamber in $\tilde{X}_{1}^{n}$, then the same statement holds for the objects bijectively associated to them.

Next we observe that the boundaries at infinity $\partial^{\infty} \tilde{X}_{i}^{n}$ also come equipped with an action of $\Gamma_{i}=\pi_{1}\left(X_{i}^{n}\right)$ by homeomorphisms, and that the induced homeomorphism $\phi^{\infty}: \partial^{\infty} \tilde{X}_{1}^{n} \rightarrow$ $\partial^{\infty} \tilde{X}_{2}^{n}$ is $\left(\Gamma_{1}, \Gamma_{2}\right)$-equivariant. It is easy to see that this implies that the bijection between the connected components of the $\mathcal{B}_{i}$ actually descends to a bijection between the connected components of the respective branching locis in the $X_{i}^{n}$. Similarly, we obtain a bijection between the chambers of $X_{1}^{n}$ and those of $X_{2}^{n}$. Furthermore, this pair of bijections are again compatible.

Now note that the chambers and connected components of the branching locus are actually topological spaces. We would like to ensure that the bijections we've obtained actually preserve the topology of the objects we are looking at. This is achieved by establishing that the fundamental group of the chambers (and branching locis) can be detected from the boundary at infinity. The technical statement is the following:

Lemma 3.1. The stabilizer of a connected lift of a chamber under the $\Gamma$-action by deck transformations coincides with the stabilizer of its boundary at infinity under the induced $\Gamma$-action on $\partial^{\infty} \tilde{X}^{n}$. The same statement holds for connected lifts of the branching locus.

The proof of this Lemma is not very hard, and makes use of the fact that the $\Gamma$-action on $\partial^{\infty} \tilde{X}^{n}$ exhibits sink/source dynamics. Now the $\left(\Gamma_{1}, \Gamma_{2}\right)$-equivariance of the homeomorphism between the boundaries at infinity immediately gives that the bijections between chambers (and between branching locis) preserve the respective fundamental groups. 
If $n \geq 3$, we can now apply Mostow rigidity for hyperbolic manifolds with totally geodesic boundaries (see Frigerio [Fr1]) to conclude that the chambers in bijective correspondance are actually isometric. A little more work ensures that the isometries between chambers glue together to give a global isometry from $X_{1}^{n}$ to $X_{2}^{n}$, completing the argument for Mostow rigidity when $n \geq 3$.

If $n=2$, we recall that an oriented surface with boundary is topologically determined by its fundamental group and the number of boundary components it has. Since we know that chambers in $X_{1}^{2}$ correspond bijectively to chambers in $X_{2}^{2}$, and that corresponding chambers have the same fundamental group and the same number of boundary components, we can (being a little careful) glue together the homeomorphisms between the various chambers to obtain a global homeomorphism. This gives us the desired topological rigidity result when $n=2$.

Finally, let us say a few words about diagram rigidity. Note that we can associate to each P-manifold a diagram of groups obtained as follows:

- corresponding to chambers and connected components of the branching locus we associate vertices labelled with the fundamental group of the respective object.

- corresponding to each containment of a connected component of the branching locus in a chamber, we associate a directed edge between the corresponding vertices, labelled with the morphism between fundamental groups induced by the inclusion.

The generalized Seifert-Van Kampen theorem now tells us that the fundamental group of the P-manifold is in fact the direct limit of the diagram of groups described above.

But we know that if the dimension is $n \geq 2$, any abstract isomorphism between fundamental groups of a pair of $n$-dimensional simple, thick, hyperbolic P-manifolds is in fact induced by a homeomorphism (an isometry if $n \geq 3$ ) between the respective P-manifolds. So in particular, if we have an abstract isomorphism between the direct limits of two diagrams as above, there is in fact an isomorphism between the underlying graphs of the diagrams of groups, having the property that corresponding vertices have isomorphic fundamental groups. Furthermore, the isomorphisms between the vertex groups can be chosen to be compatible with the edge morphisms (up to inner automorphisms), which is precisely the statement of diagram rigidity.

3.3. Quasi-isometric rigidity. For quasi-isometric rigidity, we will appeal to the following well-known result (see for instance the survey by Farb [Fa]):

Lemma 3.2. Let $X$ be a proper geodesic metric space, and assume that every quasi-isometry from $X$ to itself is in fact a bounded distance (in the sup norm) from an isometry. Furthermore, assume that a finitely generated group $G$ is quasi-isometric to $X$. Then there exists a cocompact lattice $\Gamma \subset I \operatorname{som}(X)$, and a finite group $F$ which fit into a short exact sequence:

$$
0 \longrightarrow F \longrightarrow G \longrightarrow \Gamma \longrightarrow 0
$$


In view of the Lemma, all we need to establish is that every quasi-isometry of $\tilde{X}^{n}$ is bounded distance from an isometry. In order to do this, we note that a quasi-isometry $f$ of $\tilde{X}^{n}$ induces a self-homeomorphism of $\partial^{\infty} \tilde{X}^{n}$. From our knowledge of the topology of $\partial^{\infty} \tilde{X}^{n}$, this implies that for every lift of a chamber in $\tilde{X}^{n}$, the quasi-isometry $f$ maps it to within finite distance of a (possibly different) lift of a chamber.

By suitably perturbing the map $f$ by a bounded amount, we can assume that the restriction of $f$ maps each lift of a chamber quasi-isometrically into the lift of another chamber. At this point, we appeal to a well known 'folk-theorem' (a rigorous proof of which can be found in [Fr2]): if $Y_{1}, Y_{2}$ are the universal covers of two compact hyperbolic manifolds with non-empty totally geodesic boundaries, and $g: Y_{1} \rightarrow Y_{2}$ is a quasi-isometry, then there is an isometry $\bar{g}: Y_{1} \rightarrow Y_{2}$ within bounded distance of $g$.

One now patches together the isometries between the various chambers to obtain that the original $f: \tilde{X}^{n} \rightarrow \tilde{X}^{n}$ is at finite distance from an isometry. There are two points to be careful with:

- we need to ensure that the isometries on the chambers do indeed glue together to give a global isometry.

- we need to make sure that the resulting isometry is at bounded distance from the map $f$ (i.e. that the bounded distance on each of the chambers is actually uniformly bounded).

But neither of these two points are very hard to establish. Applying the Fact now completes the argument for quasi-isometric rigidity.

\section{Concluding Remarks.}

We conclude this paper by pointing out to the reader that there are several natural questions which are still unanswered:

- Does topological rigidity hold for simple, thick, negatively curved P-manifolds of dimension $\geq 5$ ?

- For which classes of diagrams of groups does diagram rigidity hold?

- Does Mostow type rigidity still hold if we allow for a more complicated singular set (i.e. weaken the simplicity hypothesis)?

With respect to the last question, we point out that the structure of the singular set influences whether Mostow type rigidity holds. In [L1] a counterexample was given to Mostow rigidity in the general situation of thick hyperbolic P-manifolds (without the simplicity hypothesis).

\section{REFERENCES}

[A] F.D. Ancel, 'Resolving wild embeddings of codimension-one manifolds in manifolds of dimensions greater than 3', Special volume in honor of R. H. Bing (1914-1986), Topology Appl. 24 (1986), pp. 13-40. 
[AC] F.D. Ancel and J.W. Cannon, 'The locally flat approximation of cell-like embedding relations', Ann. of Math. (2) 109 (1979), pp. 61-86.

[B] R.H. Bing, 'Approximating surfaces by polyhedral ones', Ann. of Math. (2) 65 (1957), pp. 465-483.

[BH] M.R. Bridson and A. Haefliger, Metric spaces of non-positive curvature (Springer-Verlag, Berlin, 1999).

[Fa] B. Farb, 'The quasi-isometry classification of lattices in semisimple Lie groups, Math. Res. Lett. 4 (1997), pp. 705-717.

[Fr1] Frigerio R., 'Hyperbolic manifolds with geodesic boundary which are determined by their fundamental group', Topology Appl. 145 (2004), pp. 69-81.

[Fr2] Frigerio R., 'Commensurability of hyperbolic manifolds with geodesic boundary', preprint available on the ArXiv at http://front.math.ucdavis.edu/math.GT/0502209.

[L1] J.-F. Lafont, 'Rigidity result for certain 3-dimensional singular spaces and their fundamental groups', Geom. Dedicata 109 (2004), pp. 197-219.

[L2] J.-F. Lafont, 'Strong Jordan separation and applications to rigidity', to appear in J. London Math. Soc.

[L3] J.-F. Lafont, 'Rigidity of geometric amalgamations of free groups', preprint available on the ArXiv at http://front.math.ucdavis.edu/math.GR/0506518

[L4] J.-F. Lafont, 'Some open problems in Geometry and Topology', in preparation.

[M] G.D. Mostow, Strong rigidity of locally symmetric spaces (Princeton University Press, Princeton, N.J., 1973).

[S] W. Sierpinski, 'Un théorème sur les continus', Tohoku Math. Journ. 13 (1918), pp. 300-303.

Department of Mathematics, The Ohio State University, 100 Math Tower, 231 West 18th Ave., Columbus, OH 43210

E-mail address: jlafont@math.ohio-state.edu 